immer gröfser als Null ist, so mufs auch

$$
\int i \overline{d Q}
$$

immer gröfser seyn als Null; mit anderen Worten die Function

$$
A=2 i \bar{T},
$$

welche Maupertuis Wirkung (Action) genannt hat, ist - die Quelle der aufgenommenen Energie betreffend am Ende einer jeden Veränderung unbedingt kleiner, als zu Anfang derselben. Daher kann man den hypothetischen Satz, dafs die Entropie des Weltalls immer zunimmt (Clau sius), auch so ausdrücken: die Wirkung des Weltalls vermindert sich fortwährend.

\title{
VIII. Ueber ein Paradoxon der mechanischen
} Wärmetheorie; von $\mathcal{A}$. Ritter in Aachen.

Denkt man sich ein Kilogramm Luft (von der absoluten Temperatur $T_{1}$, vom Drucke $p_{1}$ und vom Volumen $v_{1}$ ) in einem unten geschlossenen Cylinder im Gleichgewichtszustande erhalten durch einen mit Gewichten belasteten Kolben, so erkennt man: dafs jede Hinzufügung von neuen Belastungen ein Sinken des Kolbens und eine Abnahme des Volumens der Luftmasse zur Folge haben wird. Durch allmähliche Steigerung der Belastung - wie z. B. bei Sandbelastung durch Hinzufügung eines Sandkornes nach dem anderen - würde das Volumen der Luftmasse bis auf jede beliebig kleine vorgeschriebene Gröfse $v_{2}$ vermindert werden können (wenigstens innerhalb der Gränzen, bis zu welchen das Mariotte-Gay-Lussac'sche Gesetz noch als gültig betrachtet werden darf).

Anders verhält es sich jedoch, wenn die Belastungszunahme plötzlich erfolgt. In diesem Falle wird das Vo- 
lumen niemals eine Grölse: $v^{2}<\frac{v_{1}}{3,44}$ erreichen können, wie aus der mechanischen Wärmetheorie auf folgende Weise sich ergiebt.

Wenn die Kolbenfläche der Einfachheit wegen gleich 1 Quadratmeter angenommen wird, so ist bei vorhandenem Gleichgewichtszustande stets der Druck der eingeschlossenen Luft pro Quadratmeter gleich der Kolbenbelastung zu setzen. Diese Belastung hatte anfänglich die Grölse $p_{1}$ und wird alsdann plötzlich bis auf die Grölse $p_{2}$ vermehrt. Beim Niedersinken von der Höhe $h_{1}$ bis zur Höhe $h_{2}$ verrichtet das Gewicht $p_{2}$ die mechanische Arbeit:

$$
\mathfrak{A}=p_{2}\left(h_{1}-h_{2}\right) \quad \ldots \quad . \quad .
$$

Da die Gefäfswände als undurchlässig für Wärme vorausgesetzt werden, so ist das Wärme-Aequivalent dieser Arbeitsgrölse dem hervorgebrachten Zuwachse der inneren Wärme gleich zu setzen; man erbält also die Gleichung:

$$
c\left(T_{2}-T_{1}\right)=A p_{2}\left(h_{1}-h_{2}\right) \quad \text {. . . ( (2), }
$$

in welcher: $c=0,1685$ die Wärmecapacität der Luft bei constantem Volumen, ferner: $A=\frac{1}{424}$ das Warme-Aequivalent der Arbeitseinheit, und $T_{2}$ die bei nachher eingetretenem Ruhestande erreichte End-Temperatur der Luftmasse bedeutet. Diese Temperatur $T_{2}$ entspricht dem Volumen: $v_{2}=h_{2} .1$ und dem Drucke $p_{2}$, ist also nach dem Mariotte-Gay-Lussac'schen Gesetze zu berechnen aus der Gleichung:

$$
p_{3} v_{2}=R T_{2} \text {, oder: } p_{2} h_{2}=29,27 . T_{2} \text {. . }
$$

und wenn man den hieraus für $T_{2}$ zu entnehmenden Werth in Gleichung (2) substituirt, so nimmt dieselbe für $p_{2}$ aufgelöst die folgende Form an:

$$
p_{2}=\frac{c R T_{1}}{(c+A R) h_{2}-A R h_{1}} \text {. . . . . }
$$

Indem man den Nenner dieses Ausdruckes gleich Null setzt, erhält man für dasjenige Compressionsverhältnifs, 
welchem der Werth $p_{2}=\infty$ entsprechen würde, die Bẹdingungsgleichung:

$$
\frac{h_{1}}{h_{2}}=1+\frac{c}{A R}=1+\frac{424 \cdot 0,1685}{23,27}=3,44
$$

Hieraus folgt, dafs unter den oben angenommenen Umständen der Kolben niemals bis auf eine Höhe: $h_{2}<\frac{h_{1}}{3,44}$ herabsinken kann - wie grols auch immer die Belastung $p_{2}$ gewählt werden möge.

Als Illustration und Seitenstück zu dem vorigen kann der in Fig. 1 dargestellte Fall betrachtet werden. Diese Figur veranschaulicht den Gleichgewichtszustand eines harten schweren cylindrischen Körpers (wie z. B. eines Mühlsteines, welcher durch die rasch auf einander folgenden Stölse einer zwischen seiner horizontalen Bodenfläche und dem Erdboden mit grofser Geschwindigkeit auf- und abfliegenden (vollkommen elastisch gedachten) Kugel frei im Raume schwebend erhalten wird. Wenn die Masse $(m)$ der Kugel sehr klein, und ihre Geschwindigkeit (c) sehr grofs angenommen wird, so ergiebt sich als Bedingung des Gleichgewichtes die Gleichung ${ }^{1}$ ):

$$
m c^{2}=P h \text {. . . . . . }
$$

Auf gleiche Weise erhält man für den in Fig. 2 dargestellten Gleichgewichtszustand die Bedingungsgleichung

$$
m v^{2}=(P+Q)(h-x) \text {. . . }
$$

Betrachtet man den in Fig. 1 dargestellten Gleichgewichtszustand als den gegebenen Anfangszustand, so überzeugt man sich leicht, dal's die neue Gleichgewichtslage (Fig. 2) nicht nur von der Gröfse der hinzugefügten Belastung $Q$ abhängen, sondern aufserdem von dem Gesetze, nach welchem die Belastung während des Sinkens von $P$ bis $P+Q$ zunahm; denn von diesem Gesetze wird die Gröfse der mechanischen Arbeit abhängen, welche die 1) Vergl. Ritter's "Lehrbuch der analytischen Mechanik“ $\$ .76$. 
Sohwerkraft während des Sinkens verrichtet. Es soll hier angenommen werden, dals das ganze Gewicht $Q$ mit einem Fig. 1.

Fig. 2.

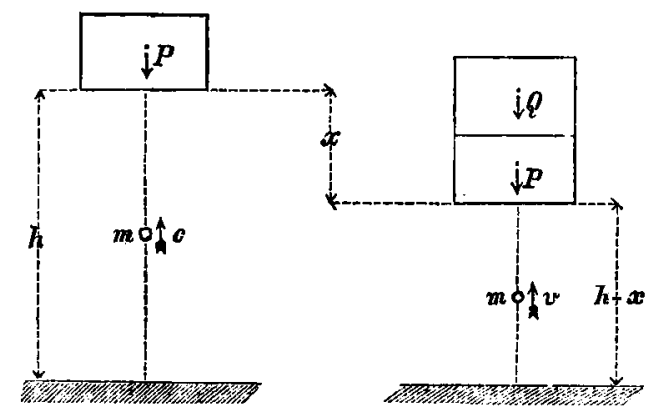

Male hinzugefügt wurde. In diesem Falle verrichtet die Schwerkraft die mechanische Arbeit: $(P+Q) x$. Diese ganze mechanische Arbeit wird - sobald die schwebende Masse nach Oscillationen von abnehmender Amplitude in der neuen Gleichgewichtslage zur Ruhe gelangt ist nunmehr in Form von lebendiger Kraft auf die oscillirende Kugel übertragen worden seyn; folglich ist:

$$
v_{2}^{2}-\frac{m c^{2}}{2}=(P+Q) x \quad . \quad \text {. }
$$

Durch Elimination der Grölsen $c$ und $v$ erbält man aus obigen drei Gleichungen für $Q$ den Werth:

$$
Q=\frac{3 P x}{h-3 x} \quad \text { • . . . . . }
$$

Indem man den Nenner dieses Ausdrucks gleich Null setzt, erhält man für diejenige Senkung, zu deren Hervorbringung ein unendlich grofses Gewicht erforderlich seyn würde, die Bedingungsgleichung:

$$
\frac{x}{h}=\frac{1}{3} \text { oder } \frac{h-x}{h}=\frac{2}{3} \quad . \quad . \quad .
$$

Es wird daher auf die hier angenommene Weise niemals ein Hinabsinken des schwebenden Körpers auf eine Höhe: $h-x<\frac{2}{3} h$ bewirkt werden können - wie grols auch immer das plötzlich hinzugefügte Uebergewicht $Q$ gewählt werden möge. 
Den Unterschied zwischen den beiden numerischen Resultaten in (5) und (10) kann man sich nach der mechanischen Theorie der Gase auf folgende Weise erklären.

Die lebendige Kraft der fortschreitenden Bewegung der Luftmolecüle beträgt: 61,5 Proc. von ihrer totalen lebendigen $\mathrm{Kraft}^{1}$ ). Die Gesammtmenge der Luftmolecüle kann man sich in drei gleiche Gruppen zerlegt denken, und annehmen, dals jede Gruppe parallel zu einer von drei rechtwinklig zu einander stehenden Coordinatenaxen sich bewegt ${ }^{2}$ ). Man findet alsdann, dafs die in verticaler Richtung sich bewegende Gruppe eine lebendige Kraft der fortschreitenden Bewegung besitzt, welche $\frac{61,5}{3}=20,5$ Proc. von der totalen lebendigen Kraft beträgt. Wenn man also in Bezug auf den in Fig. 2 dargestellten Fall die Annahme machte, dafs von der mechanischen Arbeit $(P+Q) x$ nur 20,5 Proc. auf die Vergrölserung der lebendigen Kraft der oscillirenden Kugel verwendet würden, während der Rest eine anderweitige Verwendung fände, so würde man statt Gleichung (8) die folgende Gleichung erhalten :

$$
\frac{m v^{2}}{2}-\frac{m c^{2}}{2}=0,205 \cdot(P+Q) x \quad .
$$

und als Resultat würde auch für diesen Fall sich ergeben, dafs dem Werthe $Q=\infty$ der Werth $\frac{h-x}{h}=\frac{1}{3,44}$ entspricht.

1) Vergleiche Clausius „Abhandlungen über die mechanische Stromtheorie", zweite Abhandlung, S. 258.

2) Ebendaselbst S. 251.

Aachen, den 13. Februar 1877. 